\title{
A Multiplicative Calderón Preconditioner for the Electric Field Integral Equation
}

\author{
${ }^{1}$ Francesco P. Andriulli*, ${ }^{2}$ Kristof Cools, ${ }^{1}$ Hakan Bağcı, ${ }^{2}$ Femke \\ Olyslager, ${ }^{3}$ Annalisa Buffa, ${ }^{4}$ Snorre Christiansen, and ${ }^{1}$ Eric Michielssen \\ ${ }^{1}$ University of Michigan at Ann Arbor, ${ }^{2}$ Ghent University \\ ${ }^{3}$ IMATI-CNR Pavia, ${ }^{4}$ University of Oslo
}

\section{Introduction}

A new technique for preconditioning electric field integral equations (EFIEs) by leveraging Calderón identities is presented. In contrast to all previous Calderón EFIE preconditioners, the proposed preconditioner is purely multiplicative in nature, applicable to open and closed structures, straightforward to implement, and easily interfaced with existing method of moments codes. Numerical results demonstrate that the method of moments (MoM) matrix equations obtained using the proposed preconditioner converge rapidly, independently of the discretization density.

\section{Background}

Let $\Gamma$ and $\hat{\boldsymbol{n}}_{\boldsymbol{r}}$ denote an orientable PEC surface residing in a homogeneous medium with electric permittivity $\epsilon$ and magnetic permeability $\mu$, and its outward pointing unit normal at $\boldsymbol{r}$, respectively. The current $\boldsymbol{J}(\boldsymbol{r})$ induced on $\Gamma$ by the incident electric field $\boldsymbol{E}^{i}(\boldsymbol{r})$ satisfies the EFIE

$$
\mathcal{T}(\boldsymbol{J})=-\hat{\boldsymbol{n}}_{\boldsymbol{r}} \times \boldsymbol{E}^{i} .
$$

(For the definition of $\mathcal{T}$, see [1].) To solve EFIE (1) using the MoM, $\Gamma$ is approximated by a mesh of planar triangles with minimum edge size $\delta$, and $\boldsymbol{J}(\boldsymbol{r})$ is approximated as $\boldsymbol{J}(\boldsymbol{r}) \approx \sum_{n=1}^{N} I_{n} \boldsymbol{f}_{n}(\boldsymbol{r})$ where $\boldsymbol{f}_{n}(\boldsymbol{r}), n=1, \ldots, N$ are Rao-WiltonGlisson div-conforming basis functions defined on the mesh's $N$ internal edges. To determine the coefficients $I_{n}$, the above expression for $\boldsymbol{J}(\boldsymbol{r})$ is substituted into (1) and the resulting equation is tested with curl-conforming functions $\hat{\boldsymbol{n}}_{\boldsymbol{r}} \times \boldsymbol{f}_{n}$ yielding the $N \times N$ EFIE MoM system

$$
\overline{\overline{\mathbf{Z}}} \overline{\mathbf{I}}=\overline{\mathbf{V}}
$$

where $(\overline{\overline{\mathbf{Z}}})_{i, j}=\left\langle\hat{\boldsymbol{n}}_{\boldsymbol{r}} \times \boldsymbol{f}_{i}, \mathcal{T}\left(\boldsymbol{f}_{j}\right)\right\rangle,(\overline{\mathbf{V}})_{i}=-\left\langle\hat{\boldsymbol{n}}_{\boldsymbol{r}} \times \boldsymbol{f}_{i}, \hat{\boldsymbol{n}}_{\boldsymbol{r}} \times \boldsymbol{E}^{i}\right\rangle$, and $(\overline{\mathbf{I}})_{j}=I_{j}$. For large $N,(2)$ only can be solved iteratively. Unfortunately, $\mathcal{T}$ 's singular values reside on two branches accumulating at zero and infinity, and therefore $\overline{\overline{\mathbf{Z}}}$ has a high condition number when $\delta \rightarrow 0$. Under these conditions, the iterative solution of (2) converges slowly. To solve this problem, consider the Calderón preconditioned EFIE

$$
\mathcal{T}^{2}(\boldsymbol{J})=-\mathcal{T}\left(\hat{\boldsymbol{n}}_{\boldsymbol{r}} \times \boldsymbol{E}^{i}\right) .
$$

Its discretization leads to well-conditioned EFIE MoM systems independently of $\delta$ because $\mathcal{T}^{2}$ 's singular values all reside on one branch accumulating at $-1 / 4$. Unfortunately, the direct discretization of $\mathcal{T}^{2}$ is infeasible as $\mathcal{T}\left(\boldsymbol{f}_{n}\right)$ is not available in closed-form. Invariably, bottlenecks in indirectly discretizing $\mathcal{T} \cdot \mathcal{T}$ can be traced to the need to construct a well-conditioned Gram matrix linking the domain and range of the EFIE operator. That said, a variety of methods that discretize each factor in the product $\mathcal{T}^{2}$ using ad hoc integration rules and/or operatorial manipulations exists $[2,3,4]$. Unfortunately, none of them can be implemented directly starting from an implementation of the impedance matrix $\overline{\overline{\mathbf{Z}}}$ (which is a matrix produced by any standard EFIE MoM code) and this compromises these techniques's 


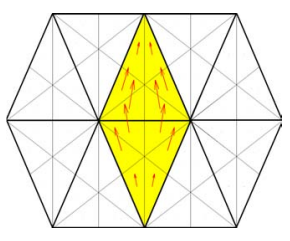

(a)

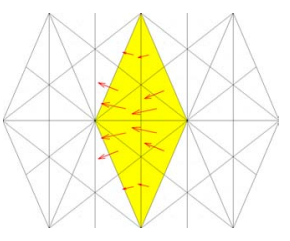

(b)

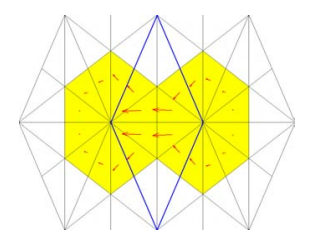

(c)

Figure 1: (a) RWG div-conforming basis function $(\boldsymbol{f})$, (b) RWG curl-conforming basis function $\left(\hat{\boldsymbol{n}}_{\boldsymbol{r}} \times \boldsymbol{f}\right)$, (c) BC quasi-curl-conforming basis function (linear combination of div-conforming RWGs defined on the barycentric mesh)

current impact on the computational electromagnetics state of the art. This paper presents a Calderón multiplicative preconditioner (CMP) that is trivially integrated into existing EFIE MoM codes. As an added advantage over existing Calderón preconditioners, the proposed preconditioner not only applies to closed structures, but (with minor modifications) to open ones as well.

\section{Calderón Multiplicative Preconditioner}

Starting from an arbitrary mesh of planar triangles that discretize $\Gamma$, further termed the initial mesh, a barycentric mesh is obtained by adding the three medians to each triangle (Fig. 1(a)); denote by $N^{b}$ the number of edges in the barycentric mesh. Note that a set of $N^{b}$ RWG basis functions $f^{b}$ can be defined on this barycentric mesh (in what follows, $\boldsymbol{f}_{i}^{b}$ will denote the RWG function defined on the $i$-th edge of the barycentric mesh). The proposed preconditioner adopts a discretization of the dual of the range of $\mathcal{T}$ on the barycentric mesh using the Buffa-Christiansen (BC) divconforming basis functions $\boldsymbol{f}_{B C}[5]$. The $\mathrm{BC}$ basis functions are defined on the edges of the initial mesh (in the following $\boldsymbol{f}_{B C i}$ will denote the $\mathrm{BC}$ function defined on the $i$-th edge of the initial mesh) and are linear combinations of div-conforming RWGs defined on the barycentric mesh (Fig. 1(c)). These functions are strictly divconforming (by construction); they also are quasi-curl-conforming in that they very much behave like curl-conforming RWGs (Fig. 1(c)). As a consequence, the Gram matrix linking BC and curl-conforming RWGs is well-conditioned since it behaves like the Gram matrix linking curl- and curl-conforming RWGs whose condition number is notoriously low [2]. These insights lead to the following discretization strategy for $\mathcal{T}^{2}=\mathcal{T} \cdot \mathcal{T}$ : the right operator $\mathcal{T}$ is discretized using div-conforming RWGs $\boldsymbol{f}$ (source) and curl-conforming RWGs $\hat{\boldsymbol{n}}_{\boldsymbol{r}} \times \boldsymbol{f}$ (test), while the left operator is discretized by using div- and quasi-curl-conforming BCs $\boldsymbol{f}_{B C}$ (source) and curl- and quasi-div-conforming BCs $\hat{\boldsymbol{n}}_{\boldsymbol{r}} \times \boldsymbol{f}_{B C}$ (test). The inverse Gram matrix between $\hat{\boldsymbol{n}}_{\boldsymbol{r}} \times \boldsymbol{f}$ and $\boldsymbol{f}_{B C}$ links the two discretizations. In other words

$$
\left(\mathcal{T}^{2}\right)_{d i s}=\overline{\overline{\mathbf{Z}}}_{B C} \overline{\overline{\mathbf{G}}}_{m}^{-1} \overline{\overline{\mathbf{Z}}}
$$

where $(\overline{\overline{\mathbf{Z}}})_{i, j}=\left\langle\hat{\boldsymbol{n}}_{\boldsymbol{r}} \times \boldsymbol{f}_{i}, \mathcal{T}\left(\boldsymbol{f}_{j}\right)\right\rangle,\left(\overline{\overline{\mathbf{Z}}}_{B C}\right)_{i, j}=\left\langle\hat{\boldsymbol{n}}_{\boldsymbol{r}} \times \boldsymbol{f}_{B C i}, \mathcal{T}\left(\boldsymbol{f}_{B C j}\right)\right\rangle$, and $\left(\overline{\overline{\mathbf{G}}}_{m}\right)_{i, j}=$ $\left\langle\hat{\boldsymbol{n}}_{\boldsymbol{r}} \times \boldsymbol{f}_{i}, \boldsymbol{f}_{B C j}\right\rangle$. The implementation of (4) can be reconducted to the computation of a single impedance matrix $\overline{\overline{\mathbf{Z}}}^{b}$ defined on the barycentric mesh, computable (and compressible) using standard codes. This is accomplished by using two transformation matrices $\overline{\overline{\mathbf{P}}} \in \mathbb{R}^{N^{b} \times N}$ and $\overline{\overline{\mathbf{R}}} \in \mathbb{R}^{N^{b} \times N}$. $\overline{\overline{\mathbf{P}}}$ 's and $\overline{\overline{\mathbf{R}}}$ 's column indices point to edges on the initial mesh, while $\overline{\overline{\mathbf{P}}}$ 's and $\overline{\overline{\mathbf{R}}}$ 's row indices point to edges 


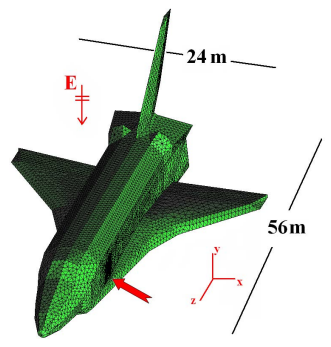

(a)

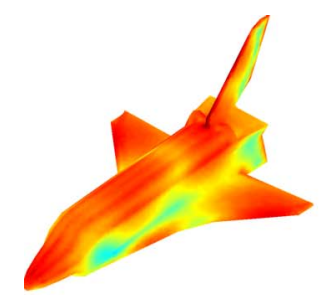

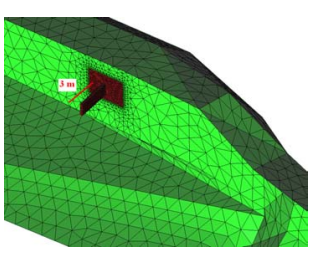

(b)

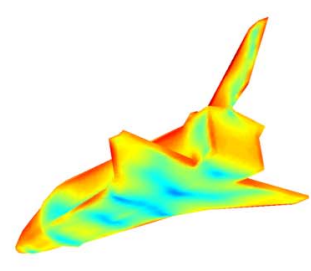

(d)

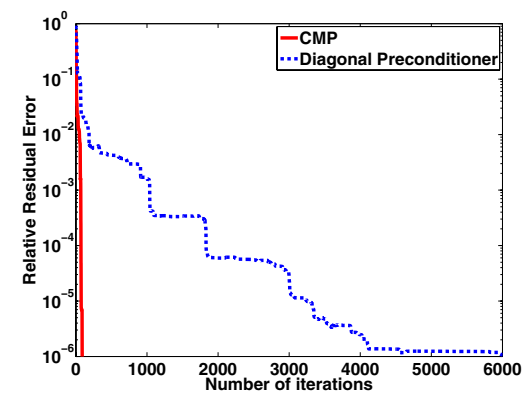

(c)

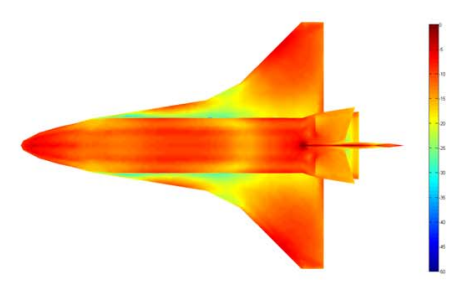

Figure 2: Analysis of scattering from a space shuttle with a waveguide antenna: (a) mesh (b) waveguide detail (c) number of iterations (d) absolute value of the current density induced on the radar dish's surface

on the barycentric one. On the $i$ th-row of $\overline{\overline{\mathbf{P}}}$ there are the coefficients of the BC basis function $\boldsymbol{f}_{B C i}$ written as a linear combination of barycentric RWG functions. Similarly, on the $i$ th-row of $\overline{\overline{\mathbf{R}}}$ there are the coefficients of the standard RWG basis function $\boldsymbol{f}_{i}$ written as a linear combination of barycentric RWG functions. The explicit expression for all these coefficients, in the case of closed and open structures, can be found in [1]. By using these matrices, equation (4), and by defining $\left(\overline{\mathbf{Z}}^{b}\right)_{i, j}=\left\langle\hat{\boldsymbol{n}}_{\boldsymbol{r}} \times \boldsymbol{f}_{i}^{b}, \mathcal{T}\left(\boldsymbol{f}_{j}^{b}\right)\right\rangle,\left(\overline{\mathbf{V}}^{b}\right)_{i}=-\left\langle\hat{\boldsymbol{n}}_{\boldsymbol{r}} \times \boldsymbol{f}_{i}^{b}, \hat{\boldsymbol{n}}_{\boldsymbol{r}} \times \boldsymbol{E}^{i}\right\rangle$, and $\overline{\overline{\mathbf{Q}}}=\overline{\overline{\mathbf{P}}} \overline{\overline{\mathbf{G}}}_{m}^{-1} \overline{\overline{\mathbf{R}}}^{T}$, it can be shown [1] that $\overline{\overline{\mathbf{Z}}}_{B C} \overline{\overline{\mathbf{G}}}_{m}^{-1} \overline{\overline{\mathbf{Z}}}=\left(\overline{\overline{\mathbf{P}}}^{T} \overline{\overline{\mathbf{Z}}}^{b} \overline{\overline{\mathbf{Q}}}_{\overline{\mathbf{Z}}}^{b} \overline{\overline{\mathbf{R}}}\right)$ and that (3) is converted into matrix equation

$$
\left(\overline{\overline{\mathbf{P}}}^{T} \overline{\overline{\mathbf{Z}}}^{b} \overline{\overline{\mathbf{Q}}} \overline{\overline{\mathbf{Z}}}^{b} \overline{\overline{\mathbf{R}}}\right) \overline{\mathbf{I}}=\left(\overline{\overline{\mathbf{P}}}^{T} \overline{\overline{\mathbf{Z}}}^{b} \overline{\overline{\mathbf{Q}}}\right) \overline{\mathbf{V}}^{b}
$$

which is the proposed CMP. The vector $\overline{\overline{\mathbf{I}}} \in \mathbb{C}^{N}$ is the same as that appearing in (2), i.e. it contains expansion coefficients for RWGs defined on the initial mesh.

\section{Numerical Results}

The performance of the proposed method is demonstrated by analyzing scattering from a space shuttle model (Fig. 2) and a radar dish (Fig. 3). Both structures are excited by $z$-polarized plane waves incident from the $y$ direction, and nonuniformly discretized; a simple scaled version of (5) is used as detailed in [1]. The shuttle is about $3 \lambda$ long at the simulation frequency $f=15 \mathrm{MHz}$. A waveguide antenna is located on one side of the shuttle's fuselage (Fig. 2(b)). The shuttle is discretized with elements that average $\lambda / 10$ in size, except of the region near the waveguide where the average element size is around $\lambda / 1200$. The number of standard RWG functions is $N=29,409$. The iterative solver required 6,032 and 89 iterations for 


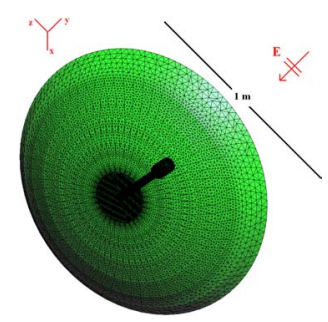

(a)

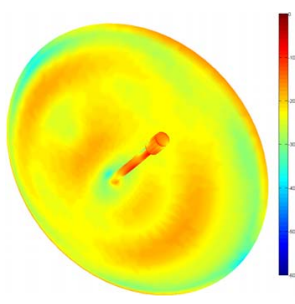

(b)

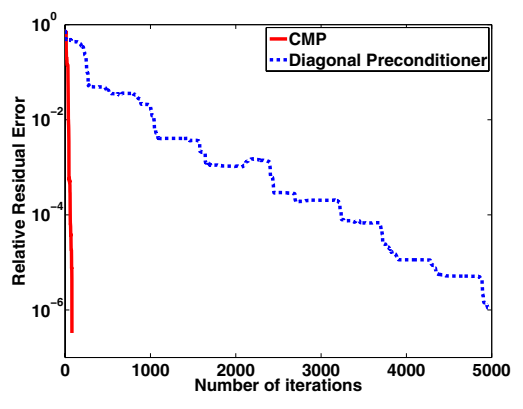

(c)

Figure 3: Analysis of scattering from radar dish: (a) mesh - note the densely populated feed- (b) absolute value of the current density induced on the radar dish's surface (c) number of iterations.

the relative residual error of the diagonally and CMP-preconditioned EFIE MoM systems' solutions to reach $10^{-6}$ (Fig. 2(c)). Fig. 2(d) shows the absolute value of the current induced on the shuttle's surface in $\mathrm{dB}$ scale from three different views. The relative norm of the difference between the solutions of the diagonally and CMP-preconditioned EFIE MoM systems is $0.1024 \%$. The radar dish diameter is $4 \lambda$ at the simulation frequency $f=1.2 \mathrm{GHz}$. The maximum and minimum edge size of the discretization are around $\lambda / 10$ and $\lambda / 650$ respectively. The number of standard RWG functions is $N=47,009$. The iterative solver required 3,251 and 60 iterations for the relative residual error of the diagonally and CMP-preconditioned EFIE MoM systems' solutions to reach $10^{-6}$ (Fig. 3(c)). Fig. 3(b) shows the absolute value of the current induced on the radar dish's surface in $\mathrm{dB}$ scale. The relative norm of the difference between the solutions of the diagonal preconditioned and CMP preconditioned EFIE MoM systems is 0.1639\%.

\section{References}

[1] F. P. Andriulli, K. Cools, H. Bagci, F. Olyslager, A. Buffa, S. Christiansen, and E. Michielssen, "A Multiplicative Calderon Preconditioner for the Electric Field Integral Equation," Accepted for publication to appear on IEEE Trans. on Antennas and Propagat., special issue on Large and Multiscale Computational Electromagnetics, 2008.

[2] R. J. Adams, "Physical and analytical properties of a stabilized electric field integral equation," IEEE Trans. Antennas Propagation, vol. 52, pp. 362-372, Feb. 2004.

[3] H. Contopanagos, B. Dembart, M. Epton, J. Ottusch, V. Rokhlin, J. Visher, and S. M. Wandzura, "Well-Conditioned Boundary Integral Equations for ThreeDimensional Electromagnetic Scattering," IEEE Trans. Antennas Propagation, vol. 50, pp. 1824-1930, Dec. 2002.

[4] S. Borel, D. P. Levadoux, and F. Alouges, "A new well-conditioned Integral formulation for Maxwell equations in three dimensions," IEEE Trans. Antennas Propagation, vol. 53, pp. 2995-3004, Sept. 2005.

[5] A. Buffa and S. Christiansen, "A dual finite element complex on the barycentric refinement," Math. Comp. , vol. 76, pp. 1743-1769, 2007. 


\section{IEEE AP-S International Symposium and \\ USNC/URSI National Radio Science Meeting}

Copyright and Reprint Permission: Abstracting is permitted with credit to the source. Libraries are permitted to photocopy beyond the limit of U.S. copyright law for private use of patrons those articles in this volume that carry a code at the bottom of the first page, provided the per-copy fee indicated in the code is paid through the Copyright Clearance Center, 222 Rosewood Drive, Danvers, MA 01923. For other copying, reprint, or replication permission, write to IEEE Copyrights Manager, IEEE Operations Center, 445 Hoes Lane, P.O. Box 1331, Piscataway, NJ, 08855-1331. All rights reserved. Copyright (c) 2008 by the Institute of Electrical and Electronics Engineers, Inc. Personal use of this material is permitted. However, permission to reprint/republish this material for advertising or promotional purposes or for creating new collective works for sale or distribution to servers or lists, or to reuse any copyrighted component of this work in other works must be obtained from the IEEE.

\section{Preliminary Version}

Support: If you have problems or questions related to the installation of this disc, please contact the 3WAIsmen at FAX: (818) 952-0183 or e-mail: wais3men@yahoo.com

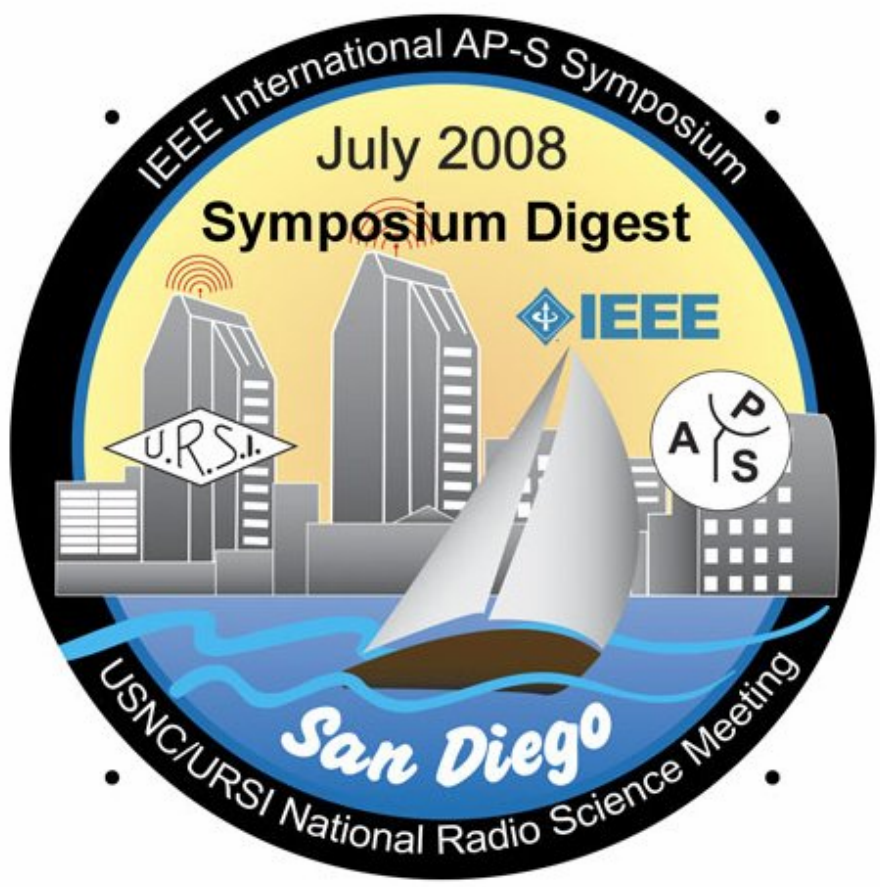

July 5-12, 2008 WAIIS $\overline{\mathbf{Z}}^{\text {mnnem }}$
San Diego, CA

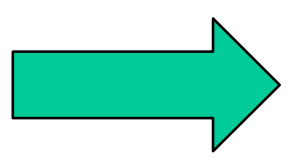

